\title{
PRENATAL EXPOSURE TO MODAFINIL ALTERS LOCOMOTOR BEHAVIOUR AND LEUCOCYTE PHAGOCYTOSIS IN MICE
}

\author{
Jana Ruda-Kucerova ${ }^{1}$, Petra Amchova ${ }^{1}$, Alena Machalova ${ }^{1}$, Jana Pistovcakova $^{1}$ \& Alexandra Sulcova ${ }^{2}$ \\ ${ }^{I}$ Department of Pharmacology, Faculty of Medicine, Masaryk University, Brno, Czech Republic \\ ${ }^{2}$ Behavioral and Social Neuroscience Group, CEITEC - Central European Institute of Technology, \\ Masaryk University, Brno, Czech Republic
}

received: 17.1.2017;

revised: 26.4.2017;

accepted: 2.7 .2017

\section{SUMMARY}

Background: Modafinil is a psychostimulant drug prescribed mainly for treatment of narcolepsy but is used as a "smart drug" by wide populations to increase wakefulness, concentration and overall mental performance. The aim of this study was to assess potential developmental toxicity of modafinil.

Materials and methods: Pregnant female mice were given either saline or modafinil (50 mg/kg orally) from gestational day (GD) 3 to GD 10 and then a challenge dose on the GD 17. The male offspring were treated analogously at the age of 10 weeks. Changes in the spontaneous locomotor/exploratory behaviour and anxiogenic profile in the open-field test were assessed in naïve animals, after an acute and 8th modafinil dose and the challenge dose following a 7-day wash-out period. One month after completion of the behavioural study, the leukocyte phagocytosis was examined by zymosan induced and luminol-aided chemiluminiscence assay in vitro.

Results: The most important finding of this study was the immunosuppressing effect on leukocyte activity, hypolocomotion and increased behavioural response to modafinil-induced psychostimulation caused by prenatal exposure to the same drug. We did not detect significantly altered anxiety-related behaviour in any group disregarding the pre-and postnatal treatments.

Conclusion: This is the first evidence of developmental toxicity of modafinil which needs to be taken into account as a potential risk factor when modafinil is administered to women who may become or are pregnant.

Key words: modafinil - prenatal administration - locomotion - phagocytosis - mice

$* * * * *$

\section{INTRODUCTION}

Modafinil is a psychostimulant drug used mainly for treatment of narcolepsy (Golicki et al. 2010), despite other indications have been proposed, e.g. as cognitive enhancer or substance abuse treatment (Mereu et al. 2013). The drug is well-tolerated and can be prescribed as a treatment of fatigue associated with AIDS (Rabkin et al. 2010) or multiple sclerosis (Tullman 2013) as well as in attention deficit hyperactivity disorder (ADHD) in children and adolescents (Wood et al. 2014). Furthermore, modafinil is used as a "smart drug" by wide populations (Vargo \& Petroczi 2016) to increase wakefulness, concentration and overall mental performance (Wood et al. 2014).

The mechanism of action is complex and probably involves numerous neurotransmitter systems. The main effect is apparently exerted via blockade of domaminergic transporter (DAT), preventing re-uptake of dopamine (DA) back to the presynaptic neuron. This effect is analogous to cocaine but weaker. Despite modafinil was shown to exert addiction (Volkow et al. 2009) it is considered a medication with low abuse potential, but the regular use is not without risk (Wisor 2013).

In preclinical studies modafinil is known to cause a robust hyperlocomotion in rodents comparable with the effect of amphetamine or methamphetamine (Simon et al. 1995). Modafinil was shown to increase locomotor activity in a dose dependent manner similarly as MDMA (3,4-methylenedioxymethamphetamine, "ecstasy") or methamphetamine but had a different ethological profile in the mouse model of agonistic behaviour. At lower than psychostimulant doses modafinil produced anxiolytic-like and antiaggressive-like effects (Machalova et al. 2010a).

On comparison with methamphetamine and MDMA which produced dose-dependent inhibition of aggression at least some doses of modafinil increased aggression and decreased timidity with no effect on sociability (Machalova et al. 2012). Also development of dependence was reported in mice although at a high (125 $\mathrm{mg} / \mathrm{kg}$ ) dose (Nguyen et al. 2011). Furthermore, D1 receptor appears to exert a primary role in modafinil-induced effects on spontaneous exploration as this effect was abolished in the D1 knock-out mice (Young et al. 2011) while D2 agonistic profile contributes to its antidepressant-like properties recently reported in a mouse model (Mahmoudi et al. 2015). Similarly as cocaine or amphetamine-like psychostimulants modafinil was shown to exert behavioural sensitization (Paterson et al. 2010, Slais et al. 2010), a phenomenon described as increased behavioural response (usually locomotor) to a repeated intermittent administration of a stable dose of addictive substance (Robinson 1984, Boutrel 2008, Watterson et al. 2016). This phenomenon may even lead to decreased drug consumption (Kucerova et al. 2009, Kucerova et al. 2012). Behavioural sensitization to drugs of abuse and the related adaptations in striatal particularly 
dopaminergic neurotransmission, are thought to play an important role in certain aspects of addiction such as tendency to relapse following abrupt drug withdrawal (Ohmori et al. 2000, Shuto et al. 2008). Pre-clinical studies use a variety of paradigms to exert this effect but they all assess the locomotor-exploratory activity at basal conditions before any treatment, after an acute drug exposure, then after a chronic treatment and lastly following a challenge dose after a period of wash-out (Landa et al. 2006, Landa et al. 2008, Paterson et al. 2010). Acute dose of a psychostimulant leads to increased locomotion and further increase after chronic exposure to the drug is considered development of sensitization. Equally high or higher locomotor response to a challenge dose is supposed to reflect expression of behavioural sensitization known to be present long after the drug discontinuation (Landa et al. 2014). In case of modafinil, the development of the sensitization was not shown but the expression after a challenge dose was observed (Paterson et al. 2010) and it is also able to induce a cross-sensitization to cocaine, i.e. increased locomotor response to a cocaine challenge dose after a chronic treatment with modafinil (Wuo-Silva et al. 2011). Modafinil as a wake-promoting agent was hypothesized to possess certain immunosuppressant effects analogously as a lack of sleep especially in patients using this drug for other than narcoleptic condition. Furthermore, a preliminary evidence shows an increase of C-reactive protein after an acute modafinil dose (Kim 2012). However, the immunomodulatory properties of modafinil have not been described in detail.

Prevalence of illicit drug use in pregnant women ranges around $4 \%$ and non-medical use of less harmful substances is expected to be even higher (Holbrook \& Rayburn 2014). Due to modafinil use as a "smart drug" (Vargo \& Petroczi 2016), the risk of its use during pregnancy is significant. Therefore, the aim of this study was to combine assessment of potential changes in the spontaneous locomotor/exploratory behaviour and anxiogenic profile in the open-field test in mice after prenatal modafinil exposure. For assessment of cell immune functions measurement of leukocyte phagocytic activity was selected (Pavelkova \& Kubala 2004). Furthermore, postnatal exposure to modafinil was evaluated in both prenatally exposed and control group and possible development of behavioural sensitization was estimated. This study may provide evidence for potentially harmful effects of modafinil when taken by pregnant women. This possibility is likely due to modafinil use for cognitive enhancing effects and moderate psychostimulation (Wood et al. 2014, Vargo \& Petroczi 2016).

\section{MATERIALS AND METHODS}

\section{Animals}

Adult male and female albino ICR mice were purchased from Masaryk University breeding facility and harem housed in cages of 5 females and 1 male (total $\mathrm{n}=30$ females and 6 males). Day 4 of the harem housing was determined as gestational day 1 (GD 1). Mice were treated by either saline (SAL, $10 \mathrm{ml} / \mathrm{kg}, \mathrm{n}=8$ ) or modafinil $(50 \mathrm{mg} / \mathrm{kg}, \mathrm{n}=7)$ from GD 3 to GD 10 and then given a challenge dose on the GD 17 (see Table 1). This schedule was repeatedly found to induce behavioural sensitization to various psychostimulants (Landa et al. 2006, Paterson et al. 2010, Landa et al. 2011, 2012). The average surviving litter size was $n=9.5$ in control and $n=10.1$ in modafinil treated mothers. No crossfostering was used, the mothers were regularly weighted and no differences were observed between control and modafinil treated mothers. The male offspring were weaned on the postnatal day (PND) 22 and housed in cages of 5 . The behavioural testing started at age 10 weeks, specifically on PND 70. The drug dosage regimen was used in the adult male offspring was analogous to the one used in mothers, known to induce behavioural sensitization (see Table 1). Thus, there were four experimental groups ( $\mathrm{n}=12$ each): SAL and MDF offspring from SAL mothers and SAL and MDF offspring from MDF treated mothers. The animals were randomly assigned to the treatment groups assuring there will be no more than 2 subjects from the same litter in every group to avoid litter effects (Holson \& Pearce 1992). Environmental conditions during the whole study were constant: relative humidity $50-60 \%$, temperature $23^{\circ} \mathrm{C}$ $\pm 1^{\circ} \mathrm{C}$, normal 12 -hour light-dark cycle (7 a.m. to 7 p.m. light). Food and water were available ad libitum. All procedures were performed in accordance with EU Directive no. 2010/63/EU and approved by the Animal Care Committee of the Faculty of Medicine, Masaryk University, Czech Republic and Czech Governmental Animal Care Committee, in compliance with Czech Animal Protection Act No. 246/1992.

\section{Drugs and treatments}

Modafinil (MDF) was isolated from a ready-made preparation (Vigil tbl. $100 \mathrm{mg}$ ) with $99.97 \%$ purity (HPLC) at the Department of Pharmaceutical Chemistry, Faculty of Pharmacy, Charles University, Czech Republic. The solution for oral gavage was prepared by suspending $50 \mathrm{mg}$ of MDF in $10 \mathrm{ml}$ of saline with $0.5 \%$ gum arabic. Care was taken to shake the suspension before use and administer a highly homogeneous material. The dose administered was $50 \mathrm{mg} / \mathrm{kg}$ at the same time in the morning hours. On the days when open-field testing was performed the administrations were done 30 minutes before the start of the test. Saline was administered as vehicle to the control groups. The oral treatment was selected due to the necessity of chronic administration to pregnant dams. The dose choice was based on the documented high sensitivity of mice (compared to rats) to modafinil (Simon et al. 1996) and behavioural effect observed after oral administration of this dose in mice (Okuro et al. 2010). We have also observed a highly significant locomotor stimulation in the mothers after an acute dose, chronic dose and the challenge dose (data not shown). 
Table 1. Study design - show treatment schedule used in mothers and their male offspring

\begin{tabular}{|c|c|c|c|c|c|c|c|c|c|c|c|c|c|c|c|}
\hline Mothers: & GD & GD & GD & GD & GD & $\begin{array}{l}\text { GD } \\
8\end{array}$ & GD & GD & GD & GD & \begin{tabular}{|l|} 
GD \\
13
\end{tabular} & GD & \begin{tabular}{|l|} 
GD \\
15
\end{tabular} & GD & GD \\
\hline $\begin{array}{c}\text { SAL } \\
(n=15)\end{array}$ & \multicolumn{8}{|c|}{ saline orally (SAL) } & \multicolumn{6}{|c|}{ No applications } & SAL \\
\hline $\begin{array}{l}\text { MDF } \\
(n=15)\end{array}$ & \multicolumn{8}{|c|}{ modafinil orally (MDF) } & \multicolumn{6}{|c|}{ No applications } & MDF \\
\hline
\end{tabular}

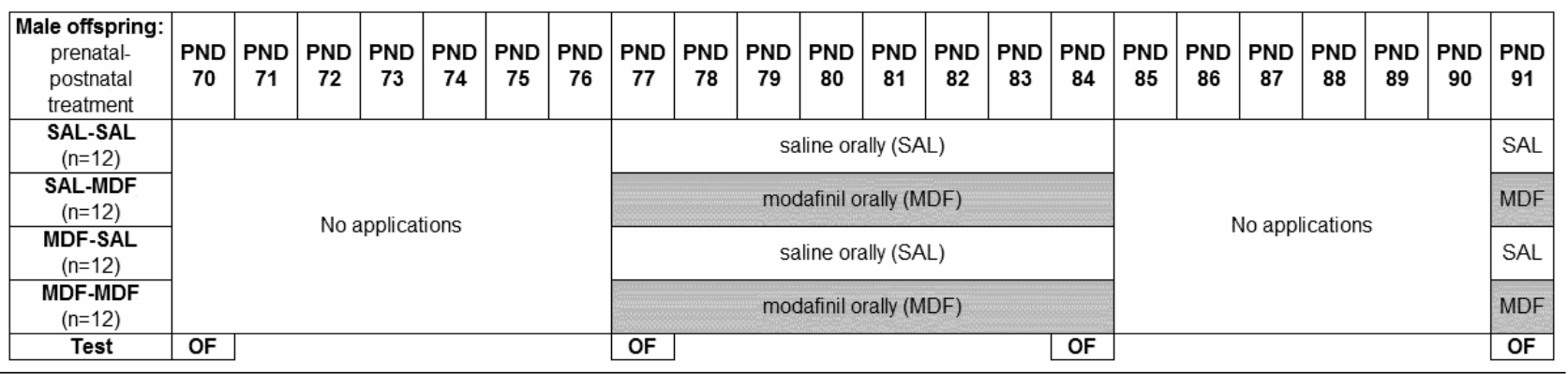

GD: gestational day; PND: postnatal day; SAL: saline treatment; MDF: modafinil treatment; OF: open-field test

\section{Locomotor activity test}

In brightly lit room, mice were individually tested for locomotor activity using the Actitrack system (Panlab, Spain) as previously described (Pistovcakova et al. 2008, Ruda-Kucerova et al. 2015, Ruda-Kucerova et al. 2016). Each plexiglass arena $(45 \times 45 \times 30 \mathrm{~cm})$ was surrounded by 2 frames equipped with photocells located one above another at 2 and $7 \mathrm{~cm}$ over the cage floor. Animals were placed individually in the centre of arena and the spontaneous horizontal (distance travelled) and vertical (rearing behaviour) locomotor activity was tracked automatically. At the end of the session, animals were returned to their home cage and the arenas were cleaned to remove potential olfactory cues. Locomotor activity (distance travelled) was recorded in the open field test for 9 minutes as follows: PND 70 - naïve mice, PND 77 - acute dose of SAL or MDF, PND 84 - one week repeated administration, PND 91 - challenge dose, i.e. acute dose after one week wash-out period. The main variables assessed were the total distance travelled as a measure of horizontal locomotion and the number of rearing episodes indicating the vertical exploratory activity. In order to assess also anxiogenic behaviour the proportion of both distance travelled and the number of rearings which took place in the central part of the arena were calculated. The central part of the arena was defined by a $10 \mathrm{~cm}$ peripheral margin from all edges.

\section{The leukocyte phagocytosis assay}

The mice were sacrificed by decapitation in short inhalation anaesthesia and trunk blood was collected into hearinized tubes. The animals were sacrificed on PND 120, i.e. one month after completion of behavioural experiment, in order to evaluate long-lasting effects of the treatments after the drug is washed-out from the system. The leukocyte phagocytosis was examined by zymosan induced and luminol-aided chemiluminiscence assay in vitro following a standard technique as described earlier (Pistovcakova et al. 2008). Briefly, $20 \mu \mathrm{l}$ of whole blood were mixed with $500 \mu \mathrm{l}$ of
Hank's solution. A $200 \mu 1$ sample was pipetted in a cuvette and $40 \mu \mathrm{l}$ of luminol (5-amino-2,3-dihydro-1,4phthalazinedione; Sigma-Aldrich, s.r.o., Prague, Czech Republic) at a concentration of $1.7 \mathrm{mg} / \mathrm{ml}$ was added. After a 10 min measurement of the "background chemiluminiscence" by the chemiluminometer (Biolumat LB 9500C, Berthold Co., Germany), phagocytosis was stimulated by addition of $40 \mu \mathrm{l}$ of opsonized zymosan. The relative degree of specific phagocytosis was calculated by extrapolating data from the standard curve and by subtracting the values for the non-specific "background". The stimulated chemiluminescence was measured at 5 min intervals during one hour. The temperature was maintained at $37^{\circ} \mathrm{C}$ throughout the whole procedure. For the measurement of systemic leukocyte counts, $20 \mu \mathrm{l}$ of blood were mixed with $10 \mathrm{ml}$ of Isotonac 3 of diluent solution in a cuvette, and 6 drops of hemolyzing reagent (Medista, s.r.o., Czech Republic) were added and mixed. One minute later, the total leukocyte count was obtained using a semi-automatic haematology analyzer MEK-5208 K (Nihon Kohden, Tokyo, Japan). For differential white blood cell counts, the blood smears were prepared immediately after blood collection using a standard coverslip technique, and were air-dried and stained with Leukodif 200 set (Bio-La-Test, Pliva-Lachema a.s., Brno, Czech Republic). Differential leukocyte counts were assessed with the help of Leukomat 5XP 83202 (Tesla Kolin k.p., Czech Republic).

\section{Statistical Data analysis}

Primary data were summarized using arithmetic mean and standard error of the mean $( \pm \mathrm{SEM})$ estimate and Kolmogorov-Smirnov test of normality was performed indicating some of the behavioural data as nonparametric. Therefore, locomotor data were analysed in every time-point by the Kruskal-Wallis ANOVA followed by multiple comparisons with Bonferroni correction. Despite some positive results of the normality test, we calculated the development of the behavioural profile by repeated measures ANOVA (factors: prenatal and postnatal treatment, repeated factor: measurement) 
Distance travelled

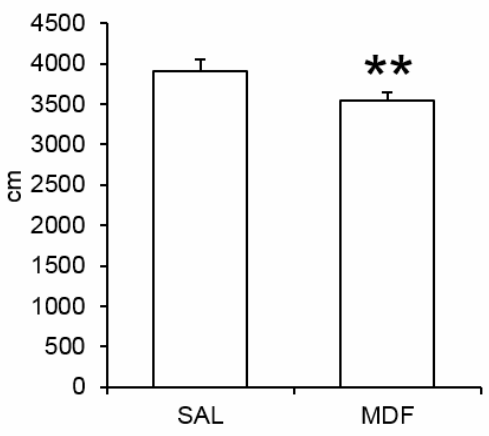

$\%$ of distance traveled in inner zone

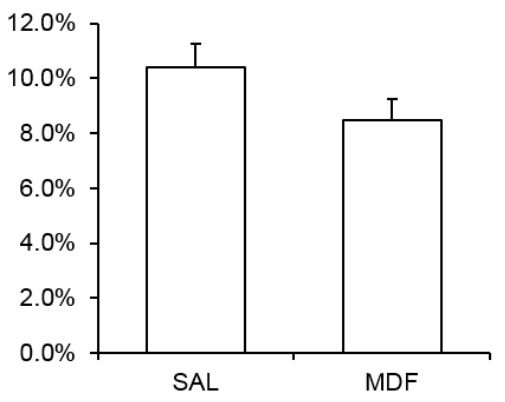

Rearings

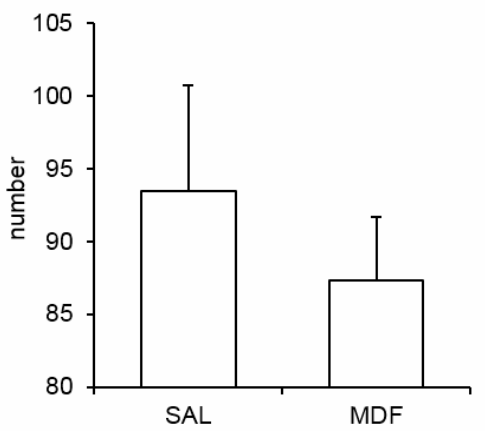

$\%$ of rearings

in inner zone

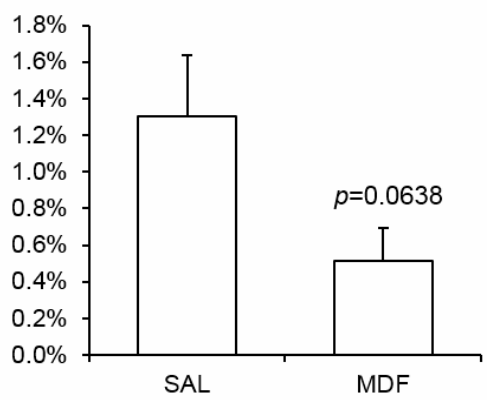

Graphs show variables measured in the open-field. The postnatal treatment was not initated at this time-point yet, therefore the animals with the same prenatal exposure are pooled ( $\mathrm{n}=24$ per group). Mann-Whitney U test revealed significantly lower distance travelled in the OF in MDF prenatally treated mice $\left({ }^{*} \mathrm{p} \leq 0.01\right)$ and a weak trend towards decrease in rearing behaviour in the inner zone of the arena in the same group. All data are shown as means \pm SEM.

Figure 1. Open-field in drug-naive animals

with Bonferroni post-hoc test, because there is no nonparametric equivalent for this comparison. The data sphericity was assessed by Mauchly test which confirmed that this assumption was not violated for any factor or interaction. The leucocyte chemiluminiscence was also analysed by repeated measures ANOVA (same factors). The analyses were calculated using Statistica 12 (StatSoft, USA). A value $\mathrm{p}<0.05$ was recognized as boundary of statistical significance in all applied tests.

\section{RESULTS}

\section{Basal open-field (PND 70)}

The basal locomotor characteristics depicted in the Figure 1 show a significant decrease of horizontal locomotion induced by the prenatal MDF exposure (MannWhitney $U$ test, $\mathrm{p}=0.005$ ). Furthermore, the proportion of rearing episodes in the central zone shows in the MDF exposed mice a trend to a decrease (Mann-Whitney $U$ test, $p=0.064$ ) suggesting a possibility of higher anxiety. In this time-point the mice were pooled depending on the prenatal exposure only.

\section{Open-field after acute dose of MDF (PND 77)}

Figure 2 pools the data obtained after the acute administration of MDF or vehicle. In this time-point the mice were already divided into 4 groups by the pre- and postnatal treatment. Interestingly, MDF did not exert a significant effect on the control animals while there was a strong stimulation in the MDF prenatally exposed group. Specifically: Kruskal-Wallis ANOVA (KW ANOVA) identified significant differences between the groups: $H(3)=22.092, p<0.001$, and test for multiple comparisons revealed a significant increase of distance travelled in the MDF-MDF mice compared with MDF-SAL $(p<0.001)$ while there was no such effect detected in the comparison of SAL-MDF and SAL-SAL mice $(p=0.265)$. A similar outcome was obtained in the rearing behaviour: KW ANOVA: $\mathrm{H}(3)=20.735, \mathrm{p}<0.001$, in multiple comparisons MDFMDF vs. MDF-SAL rendered $\mathrm{p}=0.042$. Interestingly the hypoactivity of the MDF-SAL animals (compared to SAL-SAL) was again visible in this measure: $\mathrm{p}=0.006$. No significant difference was found in the horizontal locomotion in the central zone of the arena, only the hypoactivity of the MDF-SAL animals was present analogously as in the total rearings: $\mathrm{KW}$ ANOVA: $\mathrm{H}(3)=21.045, \mathrm{p}<0.001$, multiple comparisons: MDFSAL compared to SAL-SAL, $\mathrm{p}=0.003$.

\section{Open-field after chronic exposure to MDF (PND 84)}

Chronic administration of MDF exerted similar behavioural effect as the acute one (Figure 3), i.e. higher stimulating effect on MDF prenatally exposed mice 
than the control animals. Distance travelled: $\mathrm{KW}$ ANOVA $\mathrm{H}(3)=28.621, \mathrm{p}<0.001$, and test for multiple comparisons detected a significant increase of distance travelled in the MDF-MDF mice compared to MDF-SAL
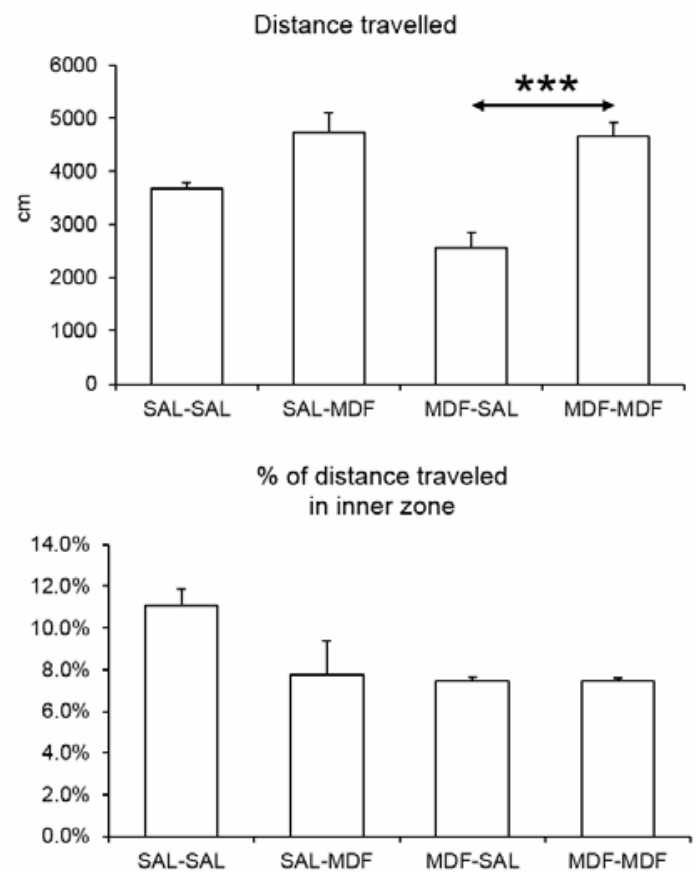

$(\mathrm{p}<0.001)$. A similar outcome was obtained in the number of rearings, KW ANOVA $\mathrm{H}(3)=21.470$, $\mathrm{p}<0.001$, MDF-MDF compared to MDF-SAL, $\mathrm{p}<0.001$. No differences among the groups were found in the central zone.

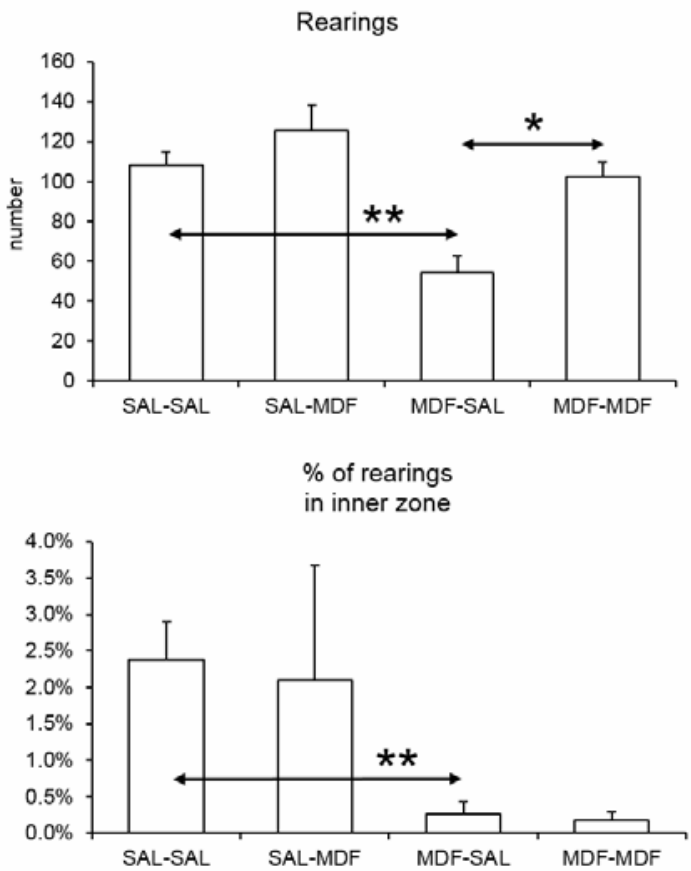

Kruskal-Wallis ANOVA indicated significant stimulating effect of MDF on both distance travelled and rearings in the MDF prenatally exposed animals only. The locomotion suppressingg effect of prenatal MDF treatment was still visible in the rearing behaviour. All data are shown as means \pm SEM, $n=12$ per group; ${ }^{*} \mathrm{p} \leq 0.05 ; \quad * * \mathrm{p} \leq 0.01 ; \quad{ }^{* * *} \mathrm{p} \leq 0.001$

Figure 2. Open-field after acute treatment
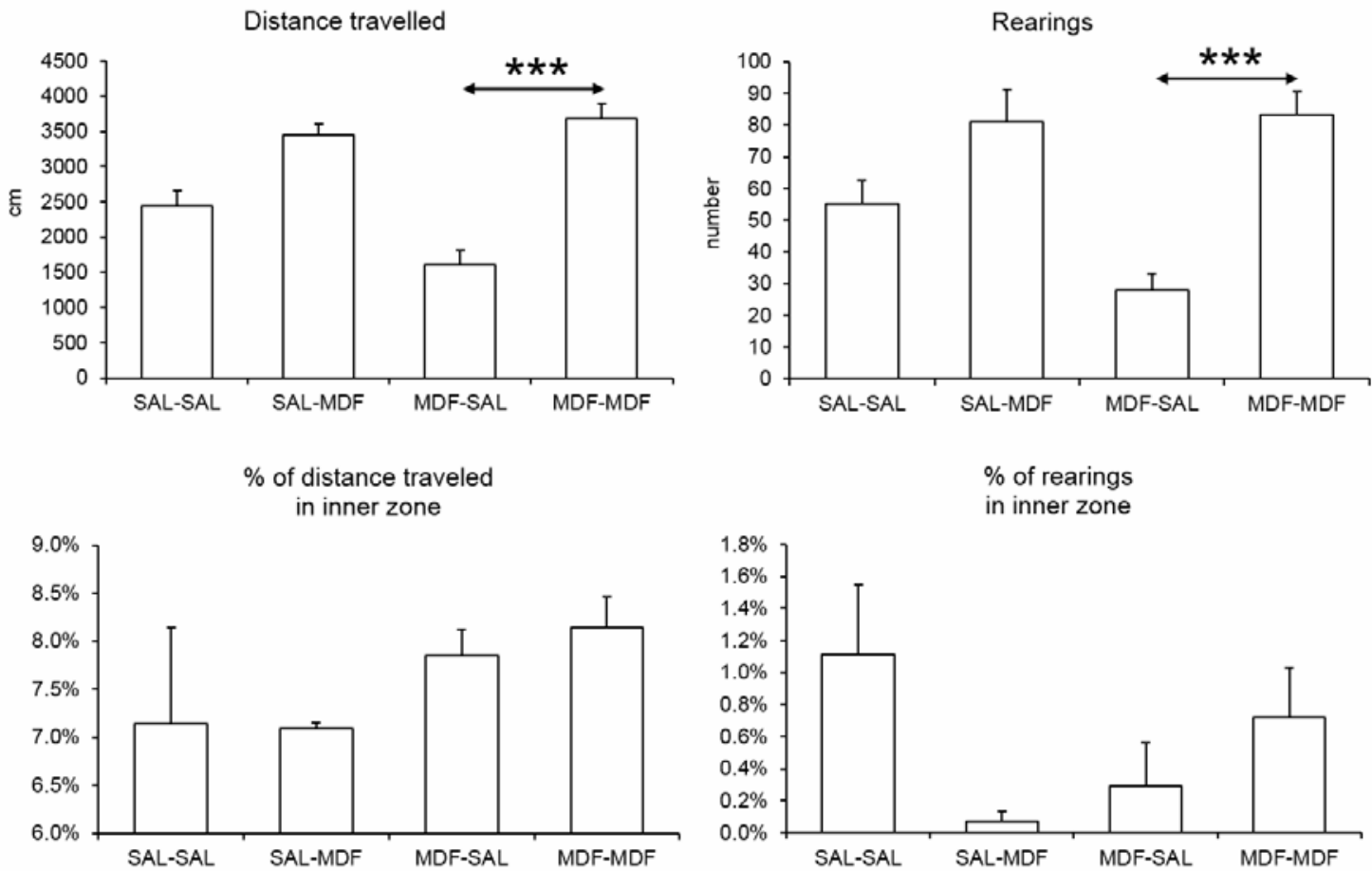

Kruskal-Wallis ANOVA indicated significant stimulating effect of MDF on both distance travelled and rearings only in the MDF prenatally exposed mice. All data are shown as means \pm SEM, $\mathrm{n}=12$ per group, ${ }^{*} \mathrm{p} \leq 0.05,{ }^{* *} \mathrm{p} \leq 0.01,{ }^{* * *} \mathrm{p} \leq 0.001$

Figure 3. Open-field after chronic treatment 

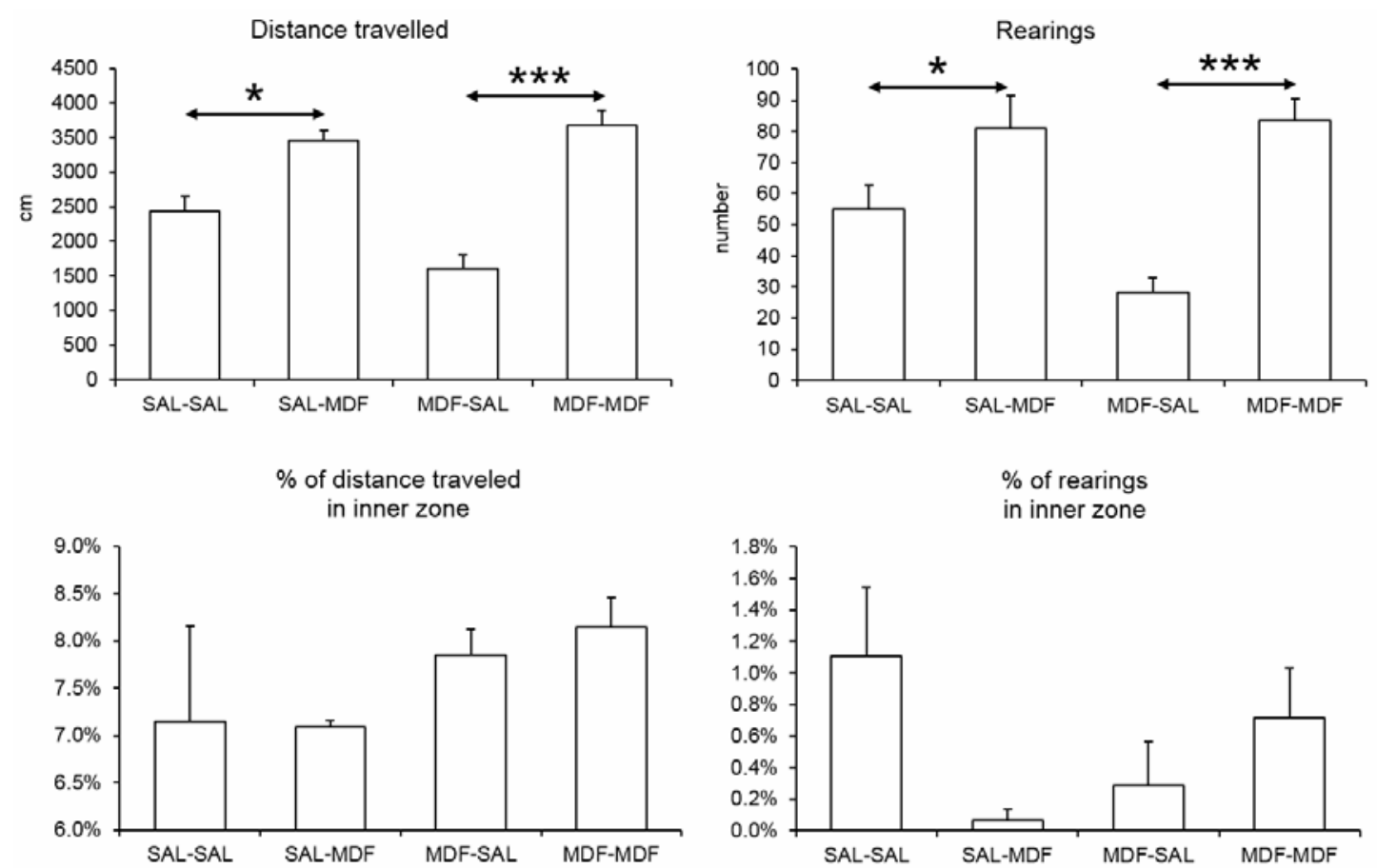

Kruskal-Wallis ANOVA indicated significant stimulating effect of MDF on both distance travelled and rearings for the first time with no regard of prenatal treatment. All data are shown as means \pm SEM, $n=12$ per group, ${ }^{*} p \leq 0.05,{ }^{* *} p \leq 0.01,{ }^{* * *} p \leq 0.001$.

Figure 4. Open-field after the challenge dose

\section{Open-field after a challenge dose of MDF (PND 91)}

The challenge MDF dose was the first to exert a significant stimulating effect on the control mice in both distance travelled and rearings. Specifically: in the distance travelled KW ANOVA showed a significant variability: $\mathrm{H}(3)=32.489, \mathrm{p}<0.001$ and multiple comparisons showed a significant differences between SALMDF and SAL-SAL mice $(p=0.011)$ as well as MDFMDF and MDF-SAL $(\mathrm{p}<0.001)$. Analogous results were observed in the rearing behaviour: $\mathrm{H}(3)=25.938$, $\mathrm{p}<0.001$, SAL-MDF vs. SAL-SAL $(\mathrm{p}=0.032)$ and MDFMDF vs. MDF-SAL ( $p=0.001)$. No differences were present in the central zone behaviours.

\section{Open-field behaviour development in time (PND 70-77-84-91)}

In order to estimate potential differences in the timecourse of development and expression of behavioural sensitization induced by repeated intermittent MDF dosing, repeated measures ANOVA (two factors: prenatal and postnatal treatment, repetition: measurements) was employed. As shown in the Figure 5, the time-dependent MDF induced changes of distance travelled were significantly dissimilar from the SAL treated animals with both types of prenatal treatment but there was no difference between them. RM ANOVA revealed significant effects of both prenatal $\left(\mathrm{F}_{(1,44)}=5.78, \mathrm{p}=0.021\right)$ and postnatal treatment $\left(\mathrm{F}_{(1,44)}=60.30, \mathrm{p}<0.001\right)$ as well as prenatal*postnatal treatment interaction $\left(\mathrm{F}_{(3,132)}=5.41\right.$, $\mathrm{p}=0.025$ ). Bonferroni post-hoc test for the prenatal*post- natal treatment interaction indicated specific differences between the groups: SAL-SAL vs. SAL-MDF, $p=0.002$ and MDF-SAL vs. MDF-MDF, $p<0.001$. Interestingly, the only difference attributable to prenatal MDF exposure was the locomotion supressing effect in the MDFSAL animals compared to SAL-SAL, Bonferroni posthoc test: $\mathrm{p}=0.010$.

In the rearing behaviour analysis, the RM ANOVA also showed significant effects of prenatal $\left(\mathrm{F}_{(1,44)}=11.14\right.$, $\mathrm{p}=0.002)$, postnatal $\left(\mathrm{F}_{(1,44)}=26.27, \mathrm{p}<0.001\right)$ and prenatal*postnatal treatment interaction $\left(\mathrm{F}_{(3,132)}=4.11\right.$, $\mathrm{p}=0.049$ ). However, Bonferroni post-hoc test indicated a significant stimulating effect of MDF only in the MDFSAL vs. MDF-MDF comparison $(p<0.001)$ but not in the SAL-SAL vs. SAL-MDF mice. Another difference attributable to prenatal MDF exposure was the rearing supressing effect in the MDF-SAL animals compared to SAL-SAL, Bonferroni post-hoc test: $\mathrm{p}=0.003$.

RM ANOVA did not indicate any significant differences in the proportion of distance travelled in the central zone. However, a significant effect of prenatal treatment $\left(F_{(1,44)}=9.17, p=0.004\right)$ was found in the number of rearing episodes in the central part of the arena, confirmed by the Bonferroni post-hoc test, $\mathrm{p}=0.004$.

\section{Leucocyte phagocytic activity (PND 120)}

For the analysis of the leucocyte chemiluminiscence data (Figure 6) a RM ANOVA indicated only a significant effect of prenatal treatment $\left(\mathrm{F}_{(1,34)}=5.57, \mathrm{p}=0.024\right)$, Bonferroni post-test, $\mathrm{p}=0.023$. This suggests that prenatal exposure to MDF may impair leucocyte phagocytic activity and consequently cause immune deficits. 

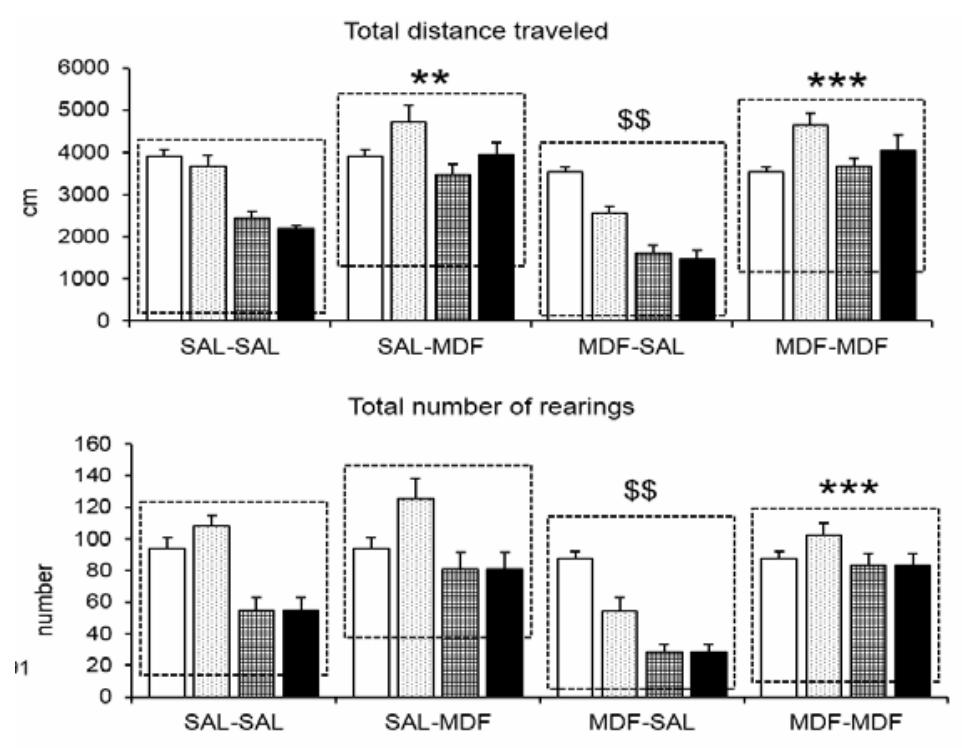

$\%$ of distance travelled in inner zone
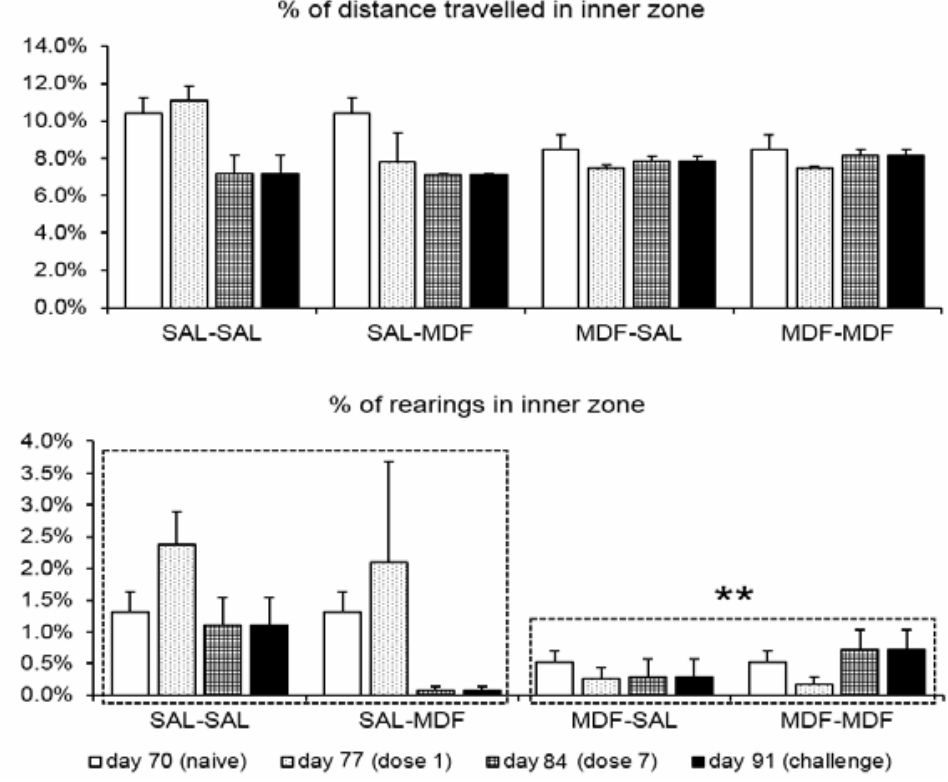

The graphs presented as means \pm SEM show the data of each group in a timeline. Repeated measures ANOVA revealed significant differences in the groups (indicated by dotted boxes) marked as: ${ }^{*} p \leq 0.05,{ }^{* *} p \leq 0.01,{ }^{* * *} \mathrm{p} \leq 0.001$ for the comparison of groups postnatally treated with vehicle and MDF with their prenatally exposed counterparts. $\$ \$ p \leq 0.01$ indicates difference between SAL-SAL and MDF-SAL animals. In the comparison of $\%$ of rearings in the centre of the arena, only prenatal treatment has a significant effect $* * p \leq 0.01$

Figure 5. Time development of open-field behaviour over the course of the study

\section{DISCUSSION}

This study revealed significant detrimental effects of prenatal modafinil exposure leading to an altered behavioural profile, different reaction to postnatal modafinil treatment and impaired leucocyte activity. The main limitation for interpretation of these results is the absence of cross-fostering in this study, i.e. it is impossible to distinguish whether the observed effects appeared due to postnatal treatment or potentially impaired rearing behaviour of the modafinil treated mothers.

Specifically, we have observed a general hypolocomotion after prenatal modafinil exposure in adult mice evaluated by the open-field test. Furthermore, an increase in behavioural indicators of psychostimulant ef- fects, i.e. distance travelled and incidence of rearing behaviour was higher in the modafinil prenatally exposed subjects. This effect was present after both acute and chronic treatment. However, it is important to note that all open-field measurements were performed under the immediate effect of the drug (30 minutes after administration). Therefore, we cannot rule out the possibility that even the reaction to the 8 th dose of the drug exerted an acute effect. Also, the fact that the prenatally exposed animals were generally hypoactive probably contributed to the significant psychostimulatory effect observed in this group. Interestingly, the challenge dose led for the first time to significant increase in both horizontal and vertical locomotor behaviour in the control animals. 


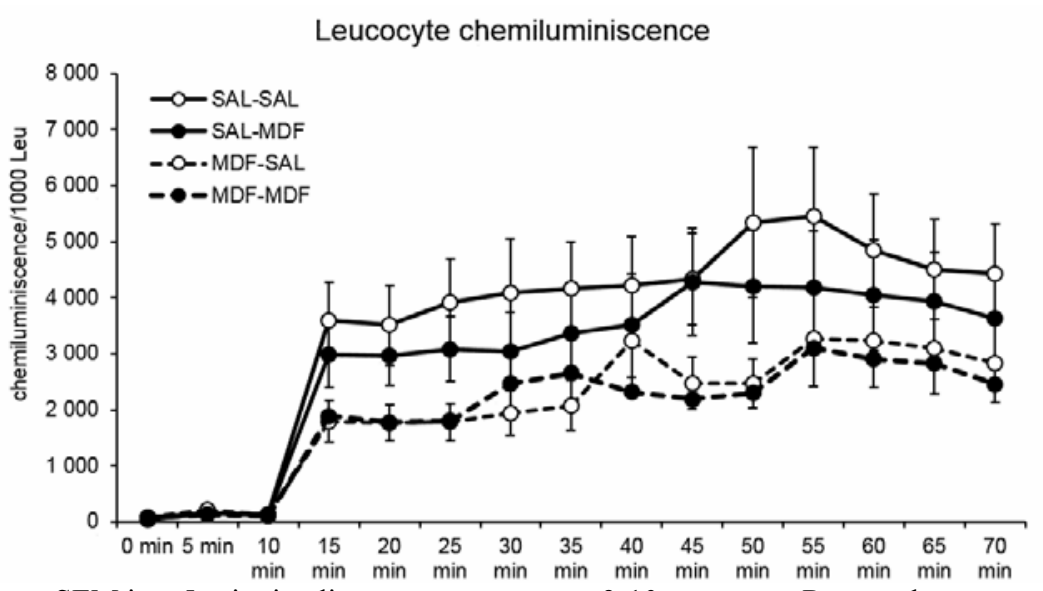

The graph shows means \pm SEM in a 5-min timeline measurements, $n=9-10$ per group. Repeated measures ANOVA with Bonferroni post-hoc test revealed significant effect of the prenatal treatment indicating immune impairment in the MDF prenatally exposed mice (SAL-SAL + SAL-MDF vs. MDF-SAL + MDF-MDF, $p=0.023$ )

Figure 6. Chemiluminiscence of phagocytes

The main behavioural effect of psychostimulants is increased locomotion (Wood et al. 2014). This was confirmed for modafinil as well (Simon et al. 1996) and is consistent with the previous finding that modafinil exerts expression but not development of behavioural sensitization (Paterson et al. 2010). Furthermore, modafinil-induced behavioural sensitization may be a prone to inter-individual variability, i.e. may develop only in some animals in the study (Soeiro Ada et al. 2012). However, when it comes to development of behavioural sensitization, classical psychostimulants are more effective. Modafinil at a dose comparable to our study $(75 \mathrm{mg} / \mathrm{kg})$ was reported to induce only a few signs of sensitization while cocaine showed robust effects (Shuman et al. 2012). This is in accordance with our data showing only a moderate tendency to develop behavioural sensitization after repeated modafinil treatment in control animals. Interestingly, prenatal exposure led to increased vulnerability to behavioural signs of psychostimulation induced by modafinil.

Psychostimulants tend to induce increased anxiety as described in caffeine (Nehlig et al. 1992), cocaine and amphetamines (Stanek 2006). In amphetamine-like drugs this effect is probably mediated via increased noradrenergic signalling which was shown to be involved in both elevated-plus maze and open-field readouts (Schmidt \& Weinshenker 2014) and modafinil is known to have agonistic effect on noradrenergic receptors alpha-1 and inhibits noradrenaline transporter (Kim 2012). Therefore, it is possible to assume modafinil has a potential to be anxiogenic. In this study we used the open-field to assess the anxiety-like behaviour by calculating the proportion of locomotion in the central part of the arena as described earlier (Royce 1977, Choleris et al. 2001). We did not observe convincing evidence of anxiogenic effect of either prenatal or postnatal exposure to modafinil. There were some trends towards decreased rearing behaviour in the central part of the arena in the prenatally exposed mice but this profile was not shown in all measurements. This is in accordance with a study where modafinil dose range 32, 64 and $128 \mathrm{mg} / \mathrm{kg}$ did not alter elevated plus behaviour (Fernandes et al. 2015). In clinical studies, only modafinil overdose was shown to be anxiogenic (Spiller et al. 2009, Carstairs et al. 2010) while clinically relevant dose in healthy volunteers lead to improved mood and no anxiety (Taneja et al. 2007). This is in accordance with our preclinical studies showing the doses without stimulant effect on locomotor behaviour in both timid and aggressive agonistic behaviour in mice the drug exhibited anxiolytic-like (selective inhibition of defensive-escape behaviour) and antiaggressive-like (suppression of aggressive acts) effects (Machalova et al. 2010b).

Therefore, we might have not observed increased anxiety due to the selection of dose. The dose of $50 \mathrm{mg} / \mathrm{kg}$ is about 5-times higher than clinically relevant and in preclinical experimentation the doses range up to $600 \mathrm{mg} / \mathrm{kg}$ (Minzenberg \& Carter 2008). In accordance with this explanation modafinil was shown to sporadically increase of noradrenaline, serotonin and dopamine in prefrontal cortex and striatum at an extreme dose of $600 \mathrm{mg} / \mathrm{kg}$, while locomotor activity was significantly raised dose-dependently after 300 and $600 \mathrm{mg} / \mathrm{kg}$ (Rowley et al. 2014).

Mechanism of action of modafinil is largely unknown but it partially shares its mechanism of action with amphetamine-like substances, although there are important differences such as the modafinil-induced dopamine transported blockade (Kim 2012). Therefore, the developmental effects of amphetamines might be similar to those induced by modafinil. Analogously as in our study with modafinil, chronic prenatal administration of methamphetamine or MDMA in rats and a challenge dose of the same drug in adulthood did not alter anxiety-related behaviour in the elevated-plus test (Macuchova et al. 2016). However, positive results were reported as well (Navarro \& Maldonado 2002, Slamberova et al. 2015). Furthermore, an open-field study revealed no effect of prenatal methamphetamine exposure in basal behavioural profile and decreased the reactivity 
to the challenge dose of the drug in adulthood (Schutova et al. 2010), which does not apply to modafinil as shown in this study. Noteworthy, some tests might be less sensitive in capturing the differences in anxiety. Elevated-plus test can render different results but social interaction test and ultrasonic vocalizations seem to be sensitive in an analogous design (Armstrong et al. 2001, Clemens et al. 2004, Slamberova et al. 2015). On the other hand, open-field test was shown to have a limited validity for anxiety-related outcome (Prut \& Belzung 2003). Therefore, our negative results on anxiety measures may be (besides dose choice) explained by the lack of sensitivity of this test. Furthermore, the issue of high methodological variability of behavioural and neurochemical approaches in developmental studies was raised recently and represents an important limitation of data interpretation (McDonnell-Dowling \& Kelly 2015, 2016).

Importantly, the assessment of leucocyte phagocytic activity revealed an impairment of this capacity in the prenatally modafinil exposed subjects but no effect of the treatment in the adult age. The central nervous system is known to modulate the immune system and sleep disturbances are known to be correlated with systemic markers of inflammation (Lorton et al. 2006). Impaired leucocyte phagocytosis was already observed in the olfactory bulbectomy model of depression (Pistovcakova et al. 2008). This model is known for multiple behavioural and neurochemical disturbances (Song \& Leonard 2005) but also disruption of the sleep architecture, specifically longer time of rapid eye movement time (Wang et al. 2012). However, acute modafinil did not produce important alterations in levels of melatonin, cortisol or growth hormone (Brun et al. 1998). Therefore, our data are in accordance showing no effect of postnatal modafinil treatment which moreover took place after a long wash-out period. Results from the pre-registration studies on reproductive and developmental toxicity revealed increased incidence in skeletal variations, embryo-foetal lethality at clinically relevant exposures and showed no teratogenic effect or impairment of growth or development of the offspring (EMA 2011). However, these studies do not include behavioural profile or immune changes. To our best knowledge this is the first report on such modafinilinduced alterations.

\section{CONCLUSION}

The most important finding of this study was the immunosuppressing effect, hypolocomotion and increased behavioural response to modafinil-induced psychostimulation caused by prenatal exposure to the same drug. We did not detect significantly altered anxietyrelated behaviour in any group with respect to the preand postnatal treatments. This is the first evidence of developmental toxicity of modafinil which needs to be taken into account as a potential risk factor when modafinil is administered to women who may become pregnant.

\section{Acknowledgements:}

This study was performed at Masaryk University as part of the project "Behavioural psychopharmacology and pharmacokinetics in preclinical drug research", number MUNI/A/1132/2017, with the support of the Specific University Research Grant, as provided by the Ministry of Education, Youth and Sports of the Czech Republic in the year 2018 and by funds from the Faculty of Medicine MU to junior researcher Jana Ruda-Kucerova.

\section{Conflict of interest: None to declare.}

\section{Contribution of individual authors:}

Jana Ruda-Kucerova was responsible for the study design, co-developed the original idea, performed literature review, and organized the experimental work. She performed the statistical analysis, prepared data for presentation and wrote the first draft of the manuscript.

Petra Amchova contributed substantially to behavioural testing and collection of data. She crosschecked the materials and methods section of the manuscript.

Alena Machalova co-developed the original idea, performed literature review and contributed to the final version of the manuscript.

Jana Pistovcakova contributed substantially to behavioural testing and collection of data and contributed to the final version of the manuscript.

Alexandra Sulcova was involved in the design of the study and discussion of the data and contributed to the final version of the manuscript.

\section{References}

1. Armstrong V, Nazarian A, Zavala AR, Krall CM, Crawford CA, McDougall SA: Effects of acute and repeated methamphetamine treatment on the ultrasonic vocalizations of postnatal rats. Pharmacol Biochem Behav 2001; 70:273-8

2. Boutrel B: A neuropeptide-centric view of psychostimulant addiction. Br J Pharmacol 2008; 154:343-57

3. Brun J, Chamba G, Kihalfallah Y, Girard P, Boissy I, Bastuji $H$, et al.: Effect of modafinil on plasma melatonin, cortisol and growth hormone rhythms, rectal temperature and performance in healthy subjects during a 36-hour sleep deprivation. J Sleep Res 1998; 7:105-14

4. Carstairs SD, Urquhart A, Hoffman J, Clark RF, Cantrell $F L$ : A retrospective review of supratherapeutic modafinil exposures. J Med Toxicol 2010; 6:307-10

5. Clemens KJ, Van Nieuwenhuyzen PS, Li KM, Cornish JL, Hunt GE, McGregor IS: Mdma ("Ecstasy"), methamphetamine and their combination: Long-term changes in social interaction and neurochemistry in the rat. Psychopharmacology (Berl) 2004; 173:318-25

6. EMA. Assessment report for modafinil containing medicinal products European Medicines Agency; 2011

7. Fernandes HA, Zanin KA, Patti Cde L, Lopes-Silva LB, Bizerra CS, Bittencourt LR, et al.: Effects of post-training modafinil administration in a discriminative avoidance task in mice. Acta Neuropsychiatr 2015; 27:235-41 
8. Golicki D, Bala MM, Niewada M, Wierzbicka A: Modafinil for narcolepsy: Systematic review and metaanalysis. Med Sci Monit 2010; 16:RA177-86

9. Holbrook BD, Rayburn WF: Teratogenic risks from exposure to illicit drugs. Obstetrics and Gynecology Clinics of North America 2014; 41:229-39

10. Holson RR, Pearce B: Principles and pitfalls in the analysis of prenatal treatment effects in multiparous species. Neurotoxicol Teratol 1992; 14:221-8

11. Choleris E, Thomas AW, Kavaliers M, Prato FS: A detailed ethological analysis of the mouse open field test: Effects of diazepam, chlordiazepoxide and an extremely low frequency pulsed magnetic field. Neurosci Biobehav Rev 2001; 25:235-60

12. Kim D: Practical use and risk of modafinil, a novel waking drug. Environ Health Toxicol 2012; 27:e2012007

13. Kucerova J, Pistovcakova J, Vrskova D, Dusek L, Sulcova $A$ : The effects of methamphetamine self-administration on behavioural sensitization in the olfactory bulbectomy rat model of depression. Int $J$ Neuropsych 2012; 15:1503-11

14. Kucerova J, Vrskova D, Sulcova A: Impact of repeated methamphetamine pretreatment on intravenous selfadministration of the drug in males and estrogenized or non-estrogenized ovariectomized female rats. Neuro Endocrinol Lett 2009; 30:663-70

15. Landa L, Jurajda M, Sulcova A: Altered cannabinoid cb1 receptor mrna expression in mesencephalon from mice exposed to repeated methamphetamine and methanandamide treatments. Neuro Endocrinol Lett 2011; 32:841-6

16. Landa L, Jurajda M, Sulcova A: Altered dopamine d1 and $d 2$ receptor mrna expression in mesencephalon from mice exposed to repeated treatments with methamphetamine and cannabinoid cb1 agonist methanandamide. Neuro Endocrinol Lett 2012; 33:446-52

17. Landa L, Machalova A, Sulcova A: Implication of nmda receptors in behavioural sensitization to psychostimulants: A short review. Eur J Pharmacol 2014; 730:77-81

18. Landa L, Slais K, Sulcova A: Impact of cannabinoid receptor ligands on sensitization to methamphetamine effects on rat locomotor behaviour. Acta Veterinaria Brno 2008; 77:183-91

19. Landa L, Sulcova A, Slais K: Involvement of cannabinoid $c b 1$ and $c b 2$ receptor activity in the development of behavioural sensitization to methamphetamine effects in mice. Neuro Endocrinol Lett 2006; 27:63-9

20. Lorton D, Lubahn CL, Estus C, Millar BA, Carter JL, Wood CA, et al.: Bidirectional communication between the brain and the immune system: Implications for physiological sleep and disorders with disrupted sleep. Neuroimmunomodulation 2006; 13:357-74

21. Macuchova E, Sevcikova M, Hrebickova I, Nohejlova K, Slamberova R: How various drugs affect anxiety-related behavior in male and female rats prenatally exposed to methamphetamine. Int J Dev Neurosci 2016; 51:1-11

22. Mahmoudi J, Farhoudi M, Talebi M, Sabermarouf B, Sadigh-Eteghad S: Antidepressant-like effect of modafinil in mice: Evidence for the involvement of the dopaminergic neurotransmission. Pharmacol Rep 2015; 67:478-84

23. Machalova A, Slais K, Vrskova D, Sulcova A: Differential effects of modafinil, methamphetamine, and mdma on agonistic behavior in male mice. Pharmacol Biochem Behav 2012; 102:215-23
24. Machalova A, Vrskova D, Slais K, Sulcova A: Acute effects of modafinil on affective behaviour in aggressive and timid male mice. Eur Neuropsychopharmacol 2010a; 20:S2082-S283

25. Machalova A, Vrskova D, Slais K, Sulcova A: Effects of modafinil acute doses on mouse agonistic behaviour. Behav Pharmacol 2010b; 21:585-6

26. McDonnell-Dowling K, Kelly JP: Sources of variation in the design of preclinical studies assessing the effects of amphetamine-type stimulants in pregnancy and lactation. Behav Brain Res 2015; 279:87-99

27. McDonnell-Dowling K, Kelly JP: The role of oxidative stress in methamphetamine-induced toxicity and sources of variation in the design of animal studies. Curr Neuropharmacol 2016; doi: 10.2174/1570159X14666160428110329, in press

28. Mereu M, Bonci A, Newman AH, Tanda G: The neurobiology of modafinil as an enhancer of cognitive performance and a potential treatment for substance use disorders. Psychopharmacology (Berl) 2013; 229:415-34

29. Minzenberg MJ, Carter CS: Modafinil: A review of neurochemical actions and effects on cognition. Neuropsychopharmacol 2008; 33:1477-502

30. Navarro JF, Maldonado E: Acute and subchronic effects of mdma ("Ecstasy") on anxiety in male mice tested in the elevated plus-maze. Prog Neuropsychopharmacol Biol Psychiatry 2002; 26:1151-4

31. Nehlig A, Daval JL, Debry G: Caffeine and the central nervous system: Mechanisms of action, biochemical, metabolic and psychostimulant effects. Brain Res Brain Res Rev 1992; 17:139-70

32. Nguyen TL, Tian YH, You IJ, Lee SY, Jang CG: Modafinilinduced conditioned place preference via dopaminergic system in mice. Synapse 2011; 65:733-41

33. Ohmori T, Abekawa T, Ito K, Koyama T: Context determines the type of sensitized behaviour: A brief review and a hypothesis on the role of environment in behavioural sensitization. Behav Pharmacol 2000; 11:211-21

34. Okuro M, Fujiki N, Kotorii N, Ishimaru Y, Sokoloff P, Nishino S: Effects of paraxanthine and caffeine on sleep, locomotor activity, and body temperature in orexin/ataxin3 transgenic narcoleptic mice. Sleep 2010; 33:930-42

35. Paterson NE, Fedolak A, Olivier B, Hanania T, Ghavami $A$, Caldarone B: Psychostimulant-like discriminative stimulus and locomotor sensitization properties of the wake-promoting agent modafinil in rodents. Pharmacol Biochem Behav 2010; 95:449-56

36. Pavelkova M, Kubala L: Luminol-, isoluminol- and lucigenin-enhanced chemiluminescence of rat blood phagocytes stimulated with different activators. Luminescence 2004; 19:37-42

37. Pistovcakova J, Dostalek M, Sulcova A, Jezova D: Tiagabine treatment is associated with neurochemical, immune and behavioural alterations in the olfactory bulbectomized rat model of depression. Pharmacopsychiatry 2008; 41:54-9

38. Prut L, Belzung $C$ : The open field as a paradigm to measure the effects of drugs on anxiety-like behaviors: A review. Eur J Pharmacol 2003; 463:3-33

39. Rabkin JG, McElhiney MC, Rabkin R, McGrath PJ: Modafinil treatment for fatigue in hiv/aids: A randomized placebo-controlled study. J Clin Psychiatry 2010; 71:707-15

40. Robinson TE: Behavioral sensitization: Characterization of enduring changes in rotational behavior produced by 
intermittent injections of amphetamine in male and female rats. Psychopharmacology (Berl) 1984; 84:466-75

41. Rowley HL, Kulkarni RS, Gosden J, Brammer RJ, Hackett $D$, Heal DJ: Differences in the neurochemical and behavioural profiles of lisdexamfetamine methylphenidate and modafinil revealed by simultaneous dual-probe microdialysis and locomotor activity measurements in freely-moving rats. J Psychopharmacol 2014; 28:254-69

42. Royce J: On the construct validity of open-field measures. Psychol Bull 1977; 84:1098-106

43. Ruda-Kucerova J, Amchova P, Babinska Z, Dusek L, Micale $V$, Sulcova A: Sex differences in the reinstatement of methamphetamine seeking after forced abstinence in sprague-dawley rats. Front Psychiatry 2015; 6:8

44. Ruda-Kucerova J, Babinska Z, Amchova P, Stark T, Drago F, Sulcova A, et al.: Reactivity to addictive drugs in the methylazoxymethanol (mam) model of schizophrenia in male and female rats. World J Biol Psychiatry 2016; 1-14, doi: 0.1080/15622975.2016.1190032, in press

45. Shuman T, Cai DJ, Sage JR, Anagnostaras SG: Interactions between modafinil and cocaine during the induction of conditioned place preference and locomotor sensitization in mice: Implications for addiction. Behav Brain Res 2012; 235:105-12

46. Shuto T, Seeman P, Kuroiwa M, Nishi A: Repeated administration of a dopamine d1 receptor agonist reverses the increased proportions of striatal dopamine dlhigh and d2high receptors in methamphetamine-sensitized rats. Eur J Neurosci 2008; 27:2551-7

47. Schmidt KT, Weinshenker D: Adrenaline rush: The role of adrenergic receptors in stimulant-induced behaviors. Mol Pharmacol 2014; 85:640-50

48. Schutova B, Hruba L, Pometlova M, Rokyta $R$, Slamberova R: Responsiveness to methamphetamine in adulthood is altered by prenatal exposure in rats. Physiol Behav 2010; 99:381-7

49. Simon P, Hemet C, Costentin J: Analysis of stimulant locomotor effects of modafinil in various strains of mice and rats. Fundam Clin Pharmacol 1996; 10:431-5

50. Simon P, Hemet C, Ramassamy C, Costentin J: Non-amphetaminic mechanism of stimulant locomotor effect of modafinil in mice. Eur Neuropsychopharmacol 1995; 5:509-14

51. Slais K, Machalova A, Vrskova D, Sulcova A: Repeated administration of modafinil induces behavioural sensitisation in mice. Eur Neuropsychopharm 2010; 20:S609

52. Slamberova R, Pometlova M, Macuchova E, Nohejlova K, Stuchlik A, Vales K: Do the effects of prenatal exposure and acute treatment of methamphetamine on anxiety vary depending on the animal model used? Behav Brain Res 2015, $292: 361-9$
53. Soeiro Ada C, Moreira KD, Abrahao KP, Quadros IM, Oliveira MG: Individual differences are critical in determining modafinil-induced behavioral sensitization and cross-sensitization with methamphetamine in mice. Behav Brain Res 2012; 233:367-74

54. Song C, Leonard BE: The olfactory bulbectomised rat as a model of depression. Neurosci Biobehav Rev 2005; 29:627-47

55. Spiller HA, Borys D, Griffith JR, Klein-Schwartz W, Aleguas A, Sollee D, et al.: Toxicity from modafinil ingestion. Clin Toxicol (Phila) 2009; 47:153-6

56. Stanek LM: Cocaine- and amphetamine related transcript (cart) and anxiety. Peptides 2006; 27:2005-11

57. Taneja I, Haman K, Shelton RC, Robertson D: A randomized, double-blind, crossover trial of modafinil on mood. J Clin Psychopharmacol 2007; 27:76-9

58. Tullman MJ: A review of current and emerging therapeutic strategies in multiple sclerosis. Am J Manag Care 2013; 19:S21-7

59. Vargo EJ, Petroczi A: "It was me on a good day": Exploring the smart drug use phenomenon in england. Front Psychol 2016; 7:779

60. Volkow ND, Fowler JS, Logan J, Alexoff D, Zhu W, Telang $F$, et al.: Effects of modafinil on dopamine and dopamine transporters in the male human brain: Clinical implications. Jama 2009; 301:1148-54

61. Wang YQ, Tu ZC, Xu XY, Li R, Qu WM, Urade Y, et al.: Acute administration of fluoxetine normalizes rapid eye movement sleep abnormality, but not depressive behaviors in olfactory bulbectomized rats. J Neurochem 2012; 120:314-24

62. Watterson LR, Kufahl PR, Taylor SB, Nemirovsky NE, Olive MF: Sensitization to the motor stimulant effects of 3,4-methylenedioxypyrovalerone ( $m d p v$ ) and crosssensitization to methamphetamine in rats. J Drug Alcohol Res 2016; 5:pii:235967

63. Wisor J: Modafinil as a catecholaminergic agent: Empirical evidence and unanswered questions. Front Neurol 2013; 4:139

64. Wood S, Sage JR, Shuman T, Anagnostaras SG: Psychostimulants and cognition: A continuum of behavioral and cognitive activation. Pharmacol Rev 2014; 66:193-221

65. Wuo-Silva R, Fukushiro DF, Borcoi AR, Fernandes HA, Procopio-Souza R, Hollais AW, et al.: Addictive potential of modafinil and cross-sensitization with cocaine: A preclinical study. Addict Biol 2011; 16:565-79

66. Young JW, Kooistra K, Geyer MA: Dopamine receptor mediation of the exploratory/hyperactivity effects of modafinil. Neuropsychopharmacol 2011; 36:1385-96.

Correspondence:

Jana Ruda-Kucerova, MD

Masaryk University, Faculty of Medicine, Department of Pharmacology

Kamenice 5, 62500 Brno, Czech Republic

E-mail:jkucer@med.muni.cz 